\title{
Evaluation of User Comprehension of a Novel Visual Search Interface
}

\author{
Adrian O'Riordan \\ Computer Science, Sir Robert Kane Building, University College Cork, Cork, Ireland \\ a.oriordan@cs.ucc.ie
}

\begin{abstract}
Advances in Web client technology now enable a richer interface thus supporting the application of visualization to the Web search process. This paper describes a user-centred evaluation of a novel visual search interface. The interface is designed to transparently support both Boolean and frequency models of information retrieval. The interface displays queries and returned results in a single simple graphical representation called Search Sphere. In contrast to both approaches that represent only queries visually and approaches that represent only results visually we integrate queries and result sets in a single visualization. We performed a user-centred study to evaluate both search query and result set comprehension. Comprehension in this context is defined as a user's correct interpretation of a given query and also his/her ability to select relevant documents from the result set visual. The feedback of this pilot study will be used to guide the development of a second prototype consisting of a rich client visual Web search interface.
\end{abstract}

Keywords: Visual Search Interfaces, Extended Boolean Search, Query Comprehension, User-centred Evaluation

\section{INTRODUCTION}

Visual search interfaces and the visualization of information spaces are active areas of research but are not yet to be widely used for Web search. The work described here is at the intersection of the fields of usability engineering, information retrieval and information visualization. We describe a visual search interface that transparently supports various models of information seeking and retrieval. The underlying retrieval model is left implicit so that the interface will be suitable for a broad spectrum of systems and users [9]. We evaluated a specific system aspect, user comprehension, using a low-fidelity prototype [25] to gain a micro-view of the requirements for interactive visual search. A user-centred evaluation was carried out that will feed into the design of a second fully functional prototype. User-centred evaluation of information seeking systems is advocated by Marchionini as a means of tackling the user-centred paradox, the fact that we cannot know "how users can best work with systems until the systems are already built" [22]. We sought to increase understanding of how visual information presentation of the search query and results impacts on user comprehension of same. User comprehension is defined as a user's correct interpretation of a visual query representation with regard to a user's ability to select the most relevant documents from the corresponding result set visualization.

The paper is organized as follows. Section 2 contains relevant background material. Section 3 introduces the Search Sphere visualization and Section 3.1 discusses specific issues related to user comprehension of same. Section 4 contains the evaluation and suggestions for progression. Section 5 describes related work. The paper finishes with a conclusions section.

\section{BACKGROUND ON VISUAL SEARCH}

Numerous techniques have been developed for visualizing the information space of a document collection. For example using "points of interest" visualizations where forces of attraction are used to position a document's location in a display [12]. Visualization of document clusters [21][38] organise results with spatial layouts. Another approach to visual search is to explicitly show query-document associations, as in Hearst's TileBars visualization [11]. Much of this work focuses on visualizing the document collection or query results and not the querying process itself. Our work focuses on graphical querying and the related results presentation.

Graphical approaches to query formulation are an alternative to the dominant text based method. Intended benefits are tied to the properties of direct manipulation [30]. Graphical approaches attempt to reframe querying and the search process as direct manipulation. Early work on graphical querying focused on visual representations of Boolean expressions as applied to querying relational databases [23]. These ideas have also been applied to text search where Boolean queries are represented visually and again evaluated as set operations. Efforts were motivated by the difficulty users have in formulating Boolean requests textually [9][31]. Young and Shneiderman used the visual form of boxes, representing terms, arranged in sequence and parallel to represent term disjunctions and conjunction respectively [7]. A prevalent visual scheme for Boolean search has search terms 
represented as circles or ovals, akin to the sets of Venn diagrams in mathematics. In this representation, the intersection of circles corresponds to the conjunction of the corresponding terms. Note that the standard Boolean retrieval model has no provision for ranking results. This and other limitations of existent systems will be further highlighted in Section 3. VQuery is a system that implemented these ideas to provide an interface to digital libraries [17]. Shneiderman conceptualized the notion of dynamic queries, a graphical query mechanism aimed at both novices and experts [1]. Dynamic approaches to querying allow users to create, move, and remove these circles in order to formulate and reformulate queries, ideally with rapid feedback of the result sets [30].

\section{SEARCH SPHERE VISUALIZATION}

Users searching for or trying to find information are faced with a number of challenges during the search process such as precisely stating their needs and effectively using the search interface. Two of the principal tasks or activities involved are query formulation and result selection. Various and diverse models of interactive information search and seeking have been proposed [26][20] that go beyond classical information retrieval models [23,5] but all include the translation of an information need [35] into a form the machine can understand and necessitate the user interpreting the search results. We focus in particular on informational needs [7], where the intent is to acquire information assumed to be present. A query is a precise statement of an information need that a computer can process. Seach is usually an iterative process involving successive query reformulation. We took a user-centred approach in that we started design from the perspective of users' information needs and not retrieval models and carried out an early user evaluation to learn more about the requirements.

In practice, most current approaches, including the popular search engines, separate the query formulation from the display of results and results selection. In terms of screen real estate, a query (composed of search terms) is often constructed in one area, and the results displayed as a list in a separate area of the page. Search terms can be composed using query operators such as the Boolean operators. In these systems, even where results are displayed visually, as in the aforementioned KartOO visual search interface, the query is still entered in a separate search box. This separation divides user focus; eye tracking experiments of problem solving involving diagrams with separated textual annotations have found "frequent alternating fixations on specific pictorial and textual parts" [4]. This problem can be viewed as an instance of what Allen calls a user's failure of perception [3]. Thus cognitive factors influence search performance. The Search Sphere visualization was kept as simple as possible to avoid both cognitive load during user familiarization and information overload during use. This preference for a simple scheme is supported by research, for example a comprehensive meta-analysis found that "users tend to perform better with simple visual-spatial interfaces" [8].

In Search Sphere, search terms are represented as rings. Documents deemed to be relevant to a search term as shown within a ring. Currently what is displayed is an elided document name (first 25 characters). The Search Sphere contains one or more semi-transparent rings each corresponding to a search term. The terms themselves are placed at a position on the circumference of the Search Sphere, which we call the spoke which is also the centre point of its ring. Intersection of rings represents the conjunction of the corresponding terms in the query; non-intersection represents disjunction. In contrast to Venn diagram approaches such as Jones [17], results are also shown as part of the same visual representation. (In Jones' work results were displayed conventionally as a separate list.) Unlike in pure Venn diagram visualizations, these rings are not mathematical sets; the position of a document in a ring is significant and gives a measure of relevance. In Search Sphere the distance of a document's location from the corresponding spoke gives the relevance of that document to a search term. Gradient shading is used as a visual cue.

Search Sphere is not tied to any particular search system rather it is conceived as a front end to a search engine. The examples given here employed Google Search as the backend search. Multiple backend searches for the multiple queries, or points of interest [24], are required to produce the results. This is explained with an example below. Figure 1(a) shows an example with a single search term, New York. The results displayed are Google Search results where the distance of the document location from the spoke corresponds to the rank in results. The radial position is defined as the position of a document with respect to the line connecting the spoke to the centre of the Search Sphere. In this example the radial positions are not significant and where placed randomly. If a search engine supports result set clustering or document-document similarity measures this information can be used to cluster/position results in this representation. 
(a)

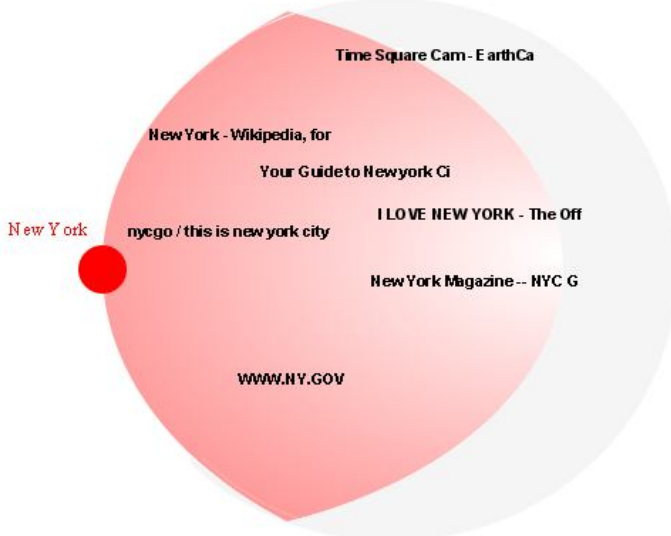

FIGURE 1: (a) Single search term and (b) Two search terms

In Figure 1(b) the first example is developed further by adding a second search term, hotel. Now you can see that results for New York U hotel, New York \hotel, and hotel $\backslash$ New York, where $U$ is the set intersection operator, and $\backslash$ the set-theoretic difference or relative complement operator. In addition, the distances of the document titles from the spokes connote information. The documents in the central intersection area are the top results returned by Google from the query "New York hotel". The relevance of documents with respect to individual search term queries could be used to position the results slighted closer to either spoke; note that this information is not readily available from Google Search. Clustering information, if available, could be utilized to place results in radial positions, but the clusters would be tempered by the need to represent distance from a spoke which should take precedence over cluster location. Spoerri argues that the lack of a strong visual cue for relevance makes it hard for users to decipher the visual maps of many document-set visualization schemes [34].

\subsection{Interpretation of Visual Metaphor}

This simple visual can express Boolean queries, term frequency approaches to search [29], hybrids of both, and also clustered results. By hybrid approaches we are referring to models of information search and retrieval that incorporate aspects of frequency models and set based (for example Boolean) search. One well-know hybrid is the p-norm Extended Boolean Model [28] which is an parameterized intermediary between the vector processing model and Boolean query processing and collapses to either depending on parameter values. Strict interpretations of Booelan queries have been found not to be compatible with the user's interpretation [28]; neither is there a provision for term weight assignment or non-binary relevance. Hence a preference for "soft" interpretations of Booean requests in system implementations. Search Sphere has no explicit parameter, catering for either interpretation depending on the underlying mechanisms.

A problem with representing each term as a circle in Venn diagram based representations is that it is awkward to represent the conjunction of more than three terms. For example, the VQuery system circumvents this by allowing the assignment of multiple query terms to a single circle [17]. While Venn diagrams can represent four or more overlapping sets, the diagrams get cluttered. We do not believe this is a major weakness since user queries tend to be very short and rarely will involve intersections of four or more terms. Search log analysis has consistently highlighted low mean query length and a low mean percentage of queries containing an intersection expression; one study gave 2.2 and 8 respectively [18]. Additionally, inventive techniques for the graphical display of more complex expression and improving visual clarity have been developed [16].

The predominant list-based representation gives few cues to support the important task of deciding on a given result's relevance besides the list ordering. (Displaying document surrogates is one way of tackling this.) In contrast, each part of the Search Sphere can be viewed to represent a different aspect of a search. For example, the results listed in the area representing the overlap of two terms relate to the logical AND of those terms. Only a small number of the most relevant results for each such aspect are displayed so as not to clutter the view causing visual crowding. We restrict displayed results to seven for each query term. We thus subdivided the problem space of visual search into two connected parts: One part of the problem is the selection of search terms and the positioning of rings to form more complex query expressions. The other part is the user interpretation of a diagram vis-à-vis comprehending the query and the corresponding map of results. We evaluated the later.

\section{EVALUATION OF USER COMPREHENSION}

We conducted an early lifecycle small-scale user study to ascertain estimates of user comprehension of Search Sphere. College undergraduate students were given search tasks to perform with the aim of determining 1) error rates and 2) preference for this type of visual search as opposed to familiar text-based search. It was the expectation that most participants were familiar with Web search. The anonymous evaluation took the form of a questionnaire that was given to undergraduate college students via prearranged contact ensuring a very high response rate. There was a time limit of 12 minutes. All of the questions were factual except for one question on 
preferences. We obtained results from 54 persons, from two disciplines: 31 from Computer Science (CS) students and 23 from Occupational Therapy (OT) students. These two groups were chosen because both would have previous exposure to Web information retrieval systems but would have used different search tools in the process.

The questions were grouped into four sections: 1) participant's search experience, 2) Google Search tasks, 3) visual search tasks, and 4) comparison of Google Search and the visual search approach. The goals of the study were presented up-front at the start. The first section contained general questions about respondents' personal profile (age, gender) and familiarity with various search engines and search methods/techniques. Section two focussed on students' ability to comprehend Google search queries of varying complexity. Boolean querying was briefly explained with examples. The Google Search interface was presented as one would see it on screen, with queries filled in for six different searches; see Figure 2(a). The six information needs for these queries were also listed but in a different order; see Figure 2(b). Participants were asked to match the queries and information needs.

(a)

\begin{tabular}{l|l|}
\hline Chicago -hotel & Google Search \\
\hline Chicago & Google Search \\
\hline Chicago hotel & Google Search \\
\hline Chicago -Hilton & Google Search \\
\hline -Chicago -hotel & Google Search \\
\hline +Chicago & Google Search \\
\hline
\end{tabular}
1. Documents about Chicago
2. Document strongly about Chicago
3. Documents about hotels in Chicago
4. Documents about Chicago excluding information about hotels
5. Documents about neither Chicago nor hotels
6. Documents about Chicago hotels but not Hilton hotels

(b)

FIGURE 2: Text search evaluation: (a) Google Search expressions and (b) Information needs

The third section introduced Search Sphere and asked the respondents to identify relevant documents for the same information needs as in section two. Included was a brief discussion of the visual metaphor. Instead of formulating queries, respondents had to choose documents that they deemed to be most relevant. The graphic in Figure 3 accompanied the questions. Note that documents were represented as simply doc1, doc2, etc. so that the titles didn't bias selection. The final section asked respondents which scheme they preferred, if any, and why.

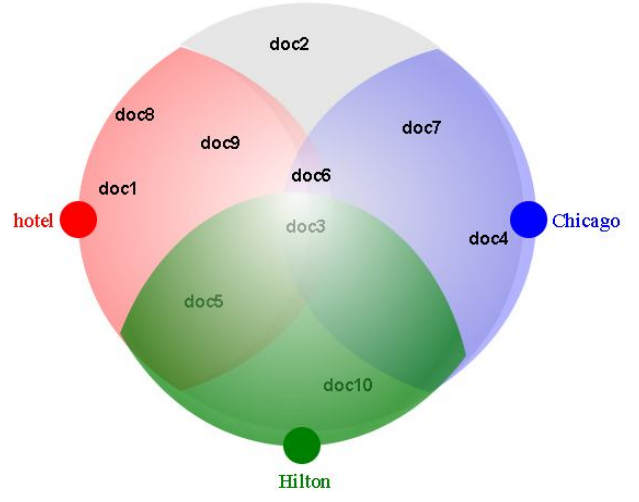

FIGURE 3: Visual search example in question

\subsection{Results and Analysis}

All the OT students had used Google Search, many Yahoo! Search and some specialized medical/health search systems (classified as non-Web Search here) but few had used advanced query techniques; see Table 1 . The numbers in Table 1 are the number of students from the total of 23 who have experience of this search engine/ type of search. The average number of correct results for the visual search was slightly higher than for Google Search; see Table 2. The numbers in columns are the numbers who got the questions correct. The normalized average is the average of column seven scaled to a maximum for 1 . The scaling allows comparison with the CS Group results below. Blank answers were interpreted as being incorrect. The low value for visual search for question six was partly down to respondents running out of time.

\begin{tabular}{cccccc}
\multicolumn{7}{c}{ TABLE 1: Search history (OT Group: 23 Total) } \\
Google Search & 23 & Yahoo! Search & 17 & Live Search & 10 \\
Any Other & 5 & Advanced Google Page & 11 & Non-Web Search & 16 \\
Boolean AND/OR & 2 & Term Exclusion & 1 & Phrasal Search & 8 \\
Other Techniques & 4 & & & & \\
\hline
\end{tabular}

TABLE 2: Correct interpretations (OT Group: 23 Total)

\begin{tabular}{ccccccccc} 
& $Q 1$ & $Q 2$ & $Q 3$ & $Q 4$ & $Q 5$ & $Q 6$ & Average & Normalized Average \\
Google & 22 & 16 & 20 & 13 & 8 & 15 & 15.7 & 0.68 \\
Visual & 18 & 20 & 14 & 16 & 18 & 9 & 15.8 & 0.69 \\
\hline
\end{tabular}


As expected the CS students had on average used more search engines and search techniques. They performed better than the OT group on the Google search tasks ( 0.75 as opposed to 0.68 for the normalized average) and they performed even better on the visual search tasks ( 0.86 as opposed to 0.69 for the normalized average). In particular both the OT and CS groups performed much better at the visual search for Q5 which contained two negations.

\begin{tabular}{cccccc}
\multicolumn{7}{c}{ TABLE 3: Search history (CS Group: 31 Total) } \\
Google Search & 31 & Yahoo! Search & 24 & Live Search & 9 \\
Any Other & 11 & Advanced Google Page & 21 & Non-Web Search & 18 \\
Boolean AND/OR & 13 & Term Exclusion & 9 & Phrase Search & 21 \\
Other Techniques & 10 & & & & \\
\hline
\end{tabular}

TABLE 4: Correct interpretations (CS Group: 31 Total)

\begin{tabular}{ccccccccc} 
& $Q 1$ & $Q 2$ & $Q 3$ & $Q 4$ & $Q 5$ & $Q 6$ & Average & Normalized Average \\
Google & 27 & 18 & 30 & 25 & 18 & 22 & 23.3 & 0.75 \\
Visual & 31 & 29 & 22 & 29 & 28 & 21 & 26.7 & 0.86 \\
\hline
\end{tabular}

Overall accuracy is higher for the visual search approach with fewer mistakes in particular when negation is involved. Both groups showed the same inclination with the difference wider for the more search-savvy CS group. These preliminary results are to feed into the development of an interactive Web search interface.

\subsection{Discussion and Second Prototype Development}

These are preliminary findings based on a mock-up prototype. In carrying out the system design and evaluation as few assumptions as possible where made about user's search behaviour. For example we did not restrict the interface or the evaluation to cater for a particular type of informational need. User studies have revealed users' search behaviour to be both diverse and unpredictable [32]. Constraining a future study to focus the examination on particular seeking tasks may elucidate more about visual search. Also the quality of a visualization is influenced by multiple factors such as expressiveness, appropriateness and effectiveness and more tests are required [6]. A second generation prototype is now being developed in Silverlight, a rich client application development environment created by Microsoft for the Web. This will enable experiments to be performed on query construction as well as comprehension. An interactive interface will allow the creation, movement and re-sizing of rings with immediate feedback of the changes in results. Cognitive research on the graphical query construction process found that two different search patterns are used to express graphical queries, neither of which is pervasive [36]. Set assembly is the classical intersecting sets in classical Venn diagrams. Set refinement is the iterative creation of successive subsets. They concluded that directly manipulable graphical interfaces that give users control over the creation and placement of sets consequently warranted investigation.

We are also interested in investigating cognitive factors such as how individual differences in visual-spatial ability may lead to performance differences in information search. Research has already shown there to be significant cognitive differences in text-based search [2]. It is highly likely that individuals will have a preferential leaning towards a particular approach. One issue in the preliminary results above is that users took longer to process the visual queries. This is an issue that will be addressed in the creation of a second prototype.

\section{RELATED WORK}

Section 5.1 reviews related usability studies of relevance and Section 5.1 summarizes emerging Web-based search visualizations.

\subsection{Visual Search Usability Studies}

Hertzum and Frokjaer [13] compared Boolean retrieval using a text-based and a Venn diagram representation and found that the later was superior in terms of mean time required to formulate a query and error rate. Grokker has a complicated visual interface which may help explain why one usability study found low user satisfaction [27]. User studies of set assembly (term disjunction) and set refinement (term conjunction, negation) in Venn diagram representations have revealed that users make fewer errors constructing queries than with text based interfaces [15]. The formulation of Boolean queries textually is surprisingly error-prone [31]. With regard to the specific issue of search results presentation, Hoeber and Yang presented a comparison of conventional list-based representations with interactive visual representations [14]. The results of this small user study indicate that users find the interactive visual approach more effective and satisfying.

\subsection{Related Visualizations}

Web technology has now developed to the point where these dynamic features can be added to a Web search interface. Rich client applications using technologies such as AJAX, Flash and Silverlight allow interaction in the browser. Many rich client search interfaces have been built in the last few years but only have a tiny share of the search market. The Flash-based KartOO (http://www.kartoo.com) is one example of a visual Web search interface but like many such systems focuses on the visualization of search results; the initial search terms are entered via a conventional search box. An example of a Web search interface that does visualize the query is Boolify 
(http://boolify.org), a Web-based educational tool enabling learners to construct Boolean queries out of puzzle pieces with a simple drag-and-drop interface. Search terms as well as AND, OR and NOT operators are represented as pieces that can be knitted together as in a jigsaw puzzle. Sortfix (http://sortfix.com/) is another search interface supporting drag-and-drop of search terms aimed at children. TwitterVenn (http://www.neoformix.com), built using the Processing programming language, allows the visualization of search terms in Twitter "tweets" as Venn diagrams. Ujiko (http://www.ujiko.com) and eyePlorer (http://www.eyeplorer.com) both use the radials of a Flash-based circle representation to guide query expansion.

The CircleSegmentViews system shares many characteristics with Search Sphere such as visual representation of AND and OR operators, use of radial information, colour coding but maps the results sets using meta-data rather than query terms. Klein and Reiterer carried out a user-centred study of visual query formation using this system [19]. Location, colour and distance are utilized in the WebStar model; a "display sphere" contains subjects which define interest centres [39]. Mooter (http://www.mooter.com) groups results in terms of themes so users can pursue the theme(s) that they are interested in. In the InfoCrystal system, in addition to supporting visual representations of Boolean queries, users can "assign relevance weights to the concepts and formulate weighted queries by interacting with a threshold slider" [33]. A system that also shares a number of features with Search Sphere is MetaCrystal [34], although MetaCrystal does not directly represent Boolean queries. With MetaCrystal a direct manipulation interface enables users to iteratively compose and edit meta-searches. MetaCrystal uses a "bull's eye" layout to enable users to visually compare the search results of multiple retrieval engines. Shape (size), colour, orientation and proximity are used to visually organize different search engine results in a circle. Whereas Search Sphere represents document relevance in terms of the distance from a spoke, MetaCrystal has a rank spiral, where most relevant results are closer to the centre. Sparkler is a visual meta-search engine using star plots where documents map to a position on a spoke based on relevance [10]. Grokker (www.groxis.com) is a visual meta-search engine that uses nested colour-coded circles or rectangles to visualize groupings of search results. Results are organized into multi-level categories. Subcategories are represented as smaller circles within their containing category. Filters can be applied, via sliders to restrict the search by source, domain, date, and information density.

\section{CONCLUSIONS}

The trend towards interactive Web interfaces will lead to increasing use of visual metaphors in search and information exploration. While research in visual user interfaces has already provided valuable lessons, more experimentation is needed to meet the requirements of visual interactive Web search. We carried out an evaluation of a novel search visualization building on work carried out previously. We assessed user query comprehension and results selection and compared against the dominant text based Web search approach as exemplified by Google Search. Results encouragingly showed that comprehension error rates were lower and preference stronger for the visual approach but the information load of processing a complex interface is a vital constraint. This will feed into the creation of a second interactive prototype which is underway.

\section{ACKNOWLEDGMENTS}

D. O'Byrne commented on a draft of the evaluation questionnaire. C. Hoare helped run the usability tests. Thanks also to F. Lane and the anonymous reviews for helpful comments.

\section{REFERENCES}

[1] Ahlberg C., Williamson, C. and Shneiderman, B. (1992) Dynamic queries for information exploration, Proceedings of the SIGCHI, Monterey, CA, 619-626.

[2] Allen, B.L. (1992) Cognitive differences in end-user searching of a CD ROM index. Proceedings of ACM SIGIR. Copenhagen, Denmark, 298-309.

[3] Allen, B.L. (1996) Information tasks - toward a user-centered approach to information systems. San Diego: Academic Press.

[4] Bertel, S. (2006) Visual focus in computer-assisted diagrammatic reasoning. In Diagrammatic Representation and Inference: LNCS 4045, 241-243. Springer Berlin, Heidelberg.

[5] Baeza-Yates, R and Ribeiro-Neto, B. (1999) Modern Information Retrieval. Addison Wesley, Harlow, England.

[6] Bederson, B.B. and Ben Shneiderman, B. (2003). The Craft of Information Visualization: Readings and Reflections. Morgan Kaufmann, San Fransisco, CA.

[7] Broder, A (2002) A taxonomy of web search. ACM SIGIR Forum, 36(2), 3-10.

[8] Chen, C. and Yu, Y. (2000) Empirical studies of information visualization: A meta-analysis. Int. J. HumanComputer Studies, 53, 851-866.

[9] Chowdhury, G.G. (2004) Modern Information Retrieval, $2^{\text {nd }}$ ed., Facet Publishing.

[10] Havre, S., Hetzler, E., Perrine, K., Jurrus, E. and Miller, N. (2001) Interactive visualization of multiple query results. Proceeding of IEEE Information Visualization Symposium, San Diego, CA.

[11] Hearst, M. (1995) TileBars: Visualization of term distribution information in full text information access, Proceedings of SIGCHI, Denver, CO, 59-66.

[12] Hemmje, M., Kunkel, C. and Willett, A. (1994) LyberWorld: A visualization user interface supporting fulltext retrieval. Proceedings of ACM SIGIR, Dublin, Ireland, 249-259. 
[13] Hertzum, M. and Frøkjær, E. (1996) Browsing and querying in online documentation: A study of user interfaces and the interaction process. ACM Transactions Computer-Human Interaction, 3(2), 136-161.

[14] Hoeber, O. and Yang, X.D. (2006) A Comparative user study of web search interfaces: HotMap, Concept Highlighter, and Google. Proceedings of Web intelligence, Washington, DC, 866-874.

[15] Jansen, B.J., Spink, A. and Saracevic, T. (2000) Real life, real users, and real needs: A study and analysis of user queries on the web. Information Processing and Management, 36, 207-227.

[16] John, C., Fish, A., Howse, J., Taylor, J. (2006) Exploring the notion of clutter in Euler diagrams, Diagrammatic Representation and Inference: LNCS 4045, 267-282.

[17] Jones, S. (1998) Dynamic query result previews for a digital library. Proceedings of ACM Conf. Digital Libraries, Pittsburgh, PA, 291-292.

[18] Jones, S., Mclnnes, S. and Staveley, M.S. (1999) A Graphical user interface for Boolean query specification. International Journal on Digital Libraries, 2(2-3), 207-223.

[19] Klein, P. and Reiterer, H. (2005) The CircleSegmentView - A visualization for query preview and visual filtering, Proc. SPIE, 5669.

[20] Kuhlthau, C.C. (2006). Kuhlthau's information search process. In K. Fisher, S. Erdelez, \& L. McKechnie (Eds.), Theories of Information Behavior, 230-234, Information Today, New Jersey.

[21] Lagus, K., Kaski, S. and Kohonen, T. (2004) Mining massive document collections by the WEBSOM method. Information Sciences, 163(1-3), 135-156.

[22] Marchionini, G. (1995) Information Seeking In Electronic Environments. Cambridge University Press, Cambridge, UK.

[23] Michard, A. (1982) Graphical presentation of Boolean expressions in a database query language: design notes and an ergonomic evaluation. Behavior and Information Technology 1(3) , 279-288.

[24] Olsen, K.A., Korfhage, R.R., Sochats, K.M., Spring, M.B. and Williams, J.G. (1993) Visualization of a document collection: The VIBE system. Information Processing and Management, 29(1), 69-81.

[25] Petrelli D. (2008) On the role of user-centred evaluation in the advancement of interactive information retrieval. Information Processing and Management, 44(1), 22-38.

[26] Pirolli, P. (2007) Information Foraging Theory: Adaptive Interaction with Information. Oxford University Press, Oxford, UK.

[27] Rivadeneira, W. and Bederson, B. (2003) A study of search result clustering interfaces: Comparing textual and zoomable user interfaces, ftp://ftp.cs.umd.edu/pub/hcil/Reports-Abstracts-Bibliography/2003-36html/2003-36.htm.

[28] Salton, G., Fox, E.A. and Wu, H. (1983) Extended Boolean information retrieval. Communications of the ACM, 26(11), 1022-1036.

[29] Salton, G. and McGill, M.J. (1986) Introduction to Modern Information Retrieval. McGraw-Hill, London, England.

[30] Shneiderman, B. (1994) Dynamic queries for visual information seeking. IEEE Software, 11(6), 70-77.

[31] Shneiderman, B. (1997) Designing the User Interface: Strategies for Effective Human-Computer Interaction. Addison-Wesley, Harlow, England.

[32] Spink, A., Wilson, T., Ellis, D., Ford, N. (1998) Modeling users' successive searches in digital environments: A National Science Foundation/British Library funded study. DLib Magazine, April 1998.

[33] Spoerri , A. (1995) InfoCrystal. Proceedings of ACM SIGIR, Seattle, WA, p. 367.

[34] Spoerri, A. (2006) Visualizing meta search results: Evaluating the MetaCrystal toolset. Proceedings of American Society for Information Science and Technology. Austin, TX.

[35] Taylor, R.S. (1962) Process of asking questions. American Documentation, 13, Oct., 391-396.

[36] Willie, S. and Bruza, P. (1995) Users' models of the information space: the case for two search models. Proceedings of ACM SIGIR, Seattle, WA, 205-210.

[37] Young, D. and Shneidermann, B. (1993) A Graphical filter/flow model for Boolean queries: An implementation and experiment. Journal of the American Society for Information Science, 44(6), 327-339.

[38] Zamir, O. and Etzioni, O. (1999) Grouper: A dynamic clustering interface to web search results. Proceedings of WWW, Toronto, Canada, 1361-1374.

[39] Zhang, J. and Nguyen, T.N. (2005) WebStar: a visualization model for hyperlink structures. Information Processing and Management, 41(4), 1003-1018. 